\title{
On the Methodology of the Analytic Method: Historical Account, Epistemological Suggestions, Stages
}

\author{
Konstantinos G. Papageorgiou \\ Post-doc researcher, \\ Department of Informatics, Ionian University. \\ University of Athens, Applied Philosophy Lab \\ email: cconstantinoss@gmail.com
}

Demetrios E. Lekkas

ex-Academic of Hellenic Open

University, Patras Greece

email: ja-dim@hotmail.com

\begin{abstract}
:
In this paper we attempt a good unearthing, sound tackling and effective pointing out of the critical key features permeating one of the two cornerstones supporting the total coordination and interplay underlying the complex epistemonic and scientific method: and that would be the constituent methodology concisely known under the cover name "analytic method". In this attempt, however, it becomes evident very soon that the direct similarities, reciprocities and complementarities to the other cornerstone, concisely known as "abstractive method", must also be laid out concurrently; the two share the same needs and behaviours, while they must also be mutually disambiguated and framed in inner consistencies and mutual contrast, to assert proper theoretical coverage, fruitful application and mutual impermeability overall.

Thus, both of them end up being discussed in parallel. To that end, simple and intuitively appealing proposals are sought, expressed and projected, regarding their key attributes, range and boundaries, both generally and especially in between them; their defining points and most essential requirements are sought out and expressed, their functional standards are suggested and the stages of its application are prescribed, according to a shadowy yet rigorous gradual breakup and assembling procedure derived from elementary combinatorics and set theory. All this gets illustrated in the schematic stages to be pursued in two relevant enlightening analytic examples, chosen so as to also partake of a crucial share of an abstractive aspect.

Two relevant issues are touched upon along the way in the vein of the present tone of quest, namely those concerning basic prerequisites of logic employed and the confusion and effective double-talk underlying the term "theory" (vs. "theories") and the consequent term-induced ambiguity in the concepts. On the subject,
\end{abstract}


analysis is the breakup of an "entity" into its "components" and synthesis is its retroactive operation, or "return", from "components" to the "entity". Similarly, "abstraction" is the course from an entity to a nesting of successive general categories, and "structure" is the other way around from more general categories towards smaller contained assemblages of entities, each uniformly exhibiting "partial manifestations" of the said categorical feature.

The semantic context and its set-theoretical implications are of paramount importance here. What is definitively taken as a "unity", in each case, is a matter of choice, and of mutual and binding agreement among the parties conducting the study and its discussion. Beyond that, figuratively speaking, the analytic method concerns "anatomical autopsies" digging into such unities constituting elements or members of sets, whereas the abstractive method concerns attributes connecting sets of such "unities" and their nested supersets and subsets. We close by sketching the stage-bystage application as illustrated in two case-studies: one from Cultural Studies, regarding a model, focusing on the primitive ternary ritual of the perfo-rming arts $[\mathrm{K} \Lambda \mathrm{M}]$, discussed complete with some key side issues, and accompanied by considerations on the triple of the material arts [X $\Psi \Omega]$, and then one from Bioethics regarding all possible partial and combinatorial constituents in consent models.

Keywords: Analytic method, Abstract method, Scientific \& Epistemonic method, Set theory, Theory, Primitive ritual, $\mathrm{K} \Lambda \mathrm{M}, \Psi \mathrm{X} \Omega$, Consent

\section{Пєрí $\eta \psi \eta$ :}

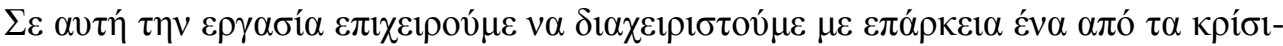

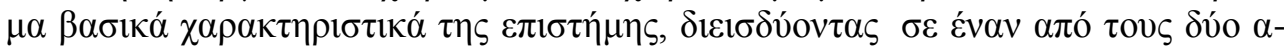

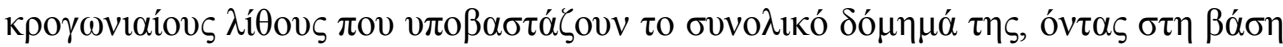

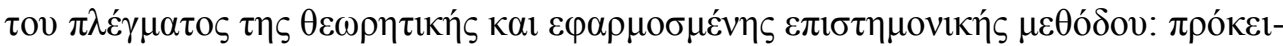

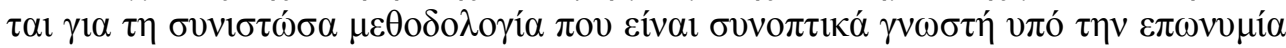

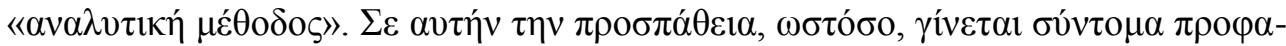

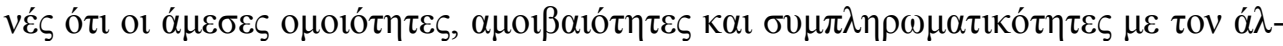

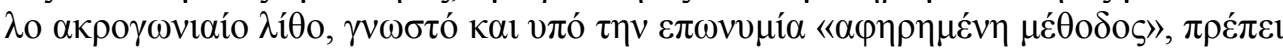

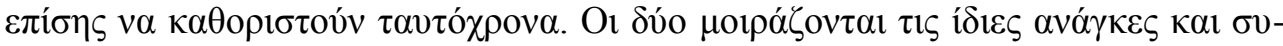

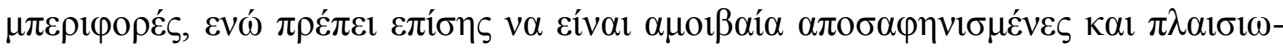

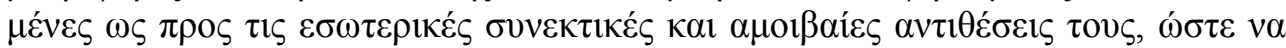

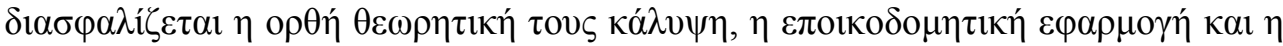

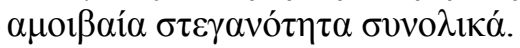

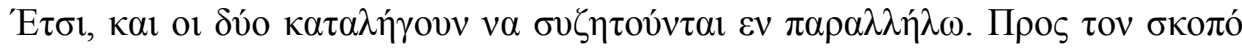

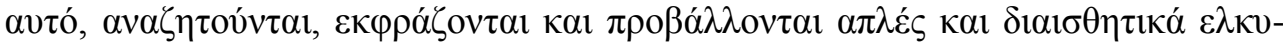

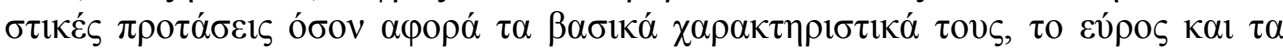

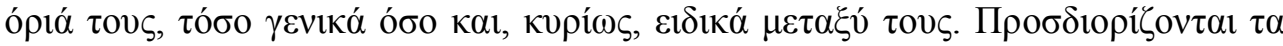

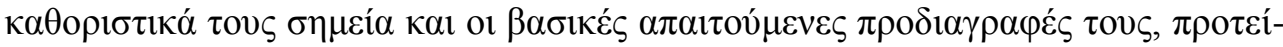

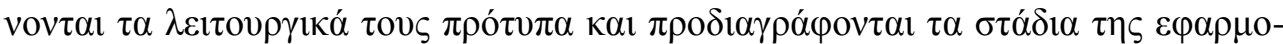

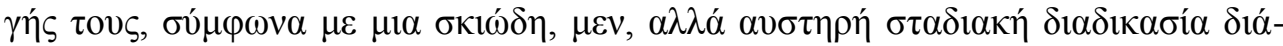

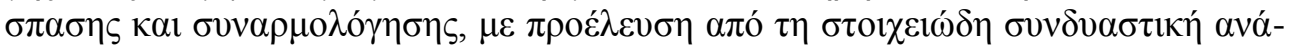




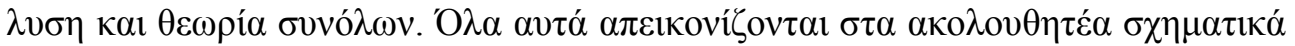

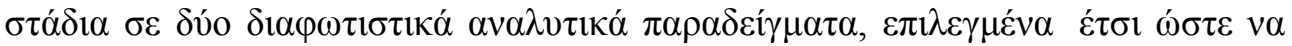

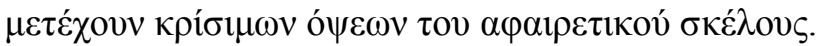

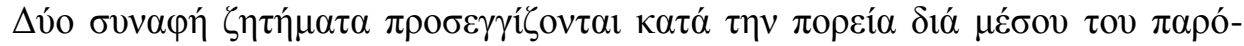

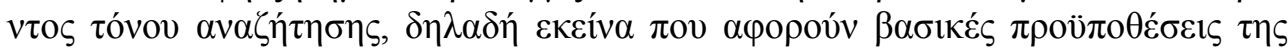

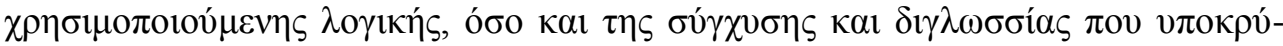

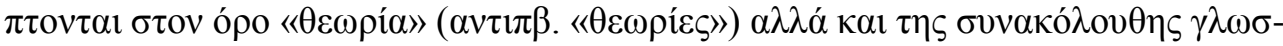

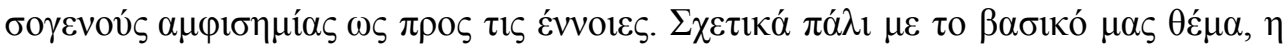

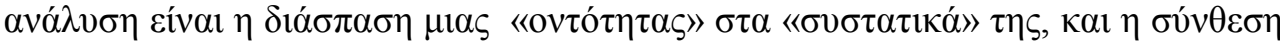

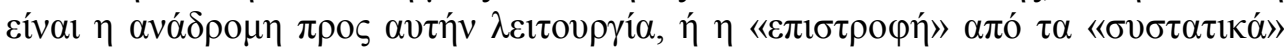

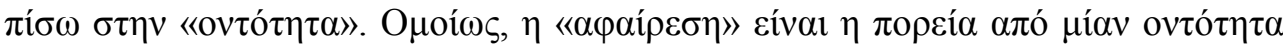

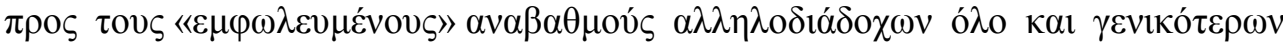

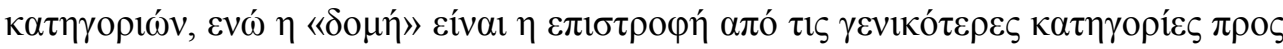

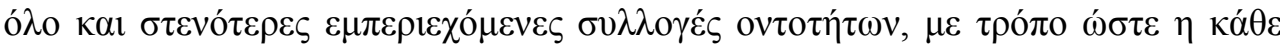

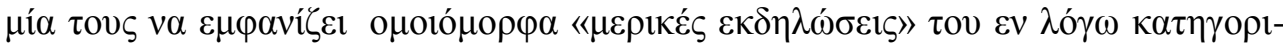

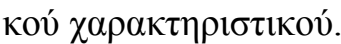

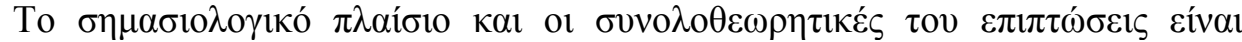

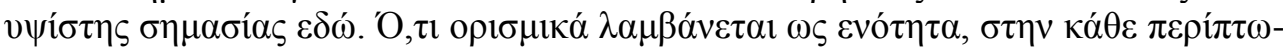

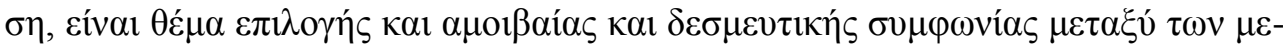

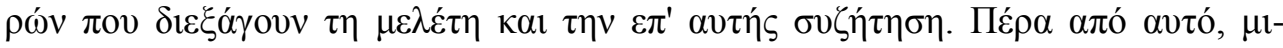

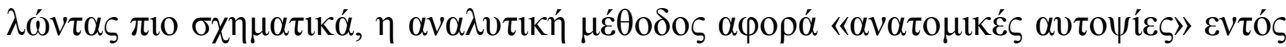

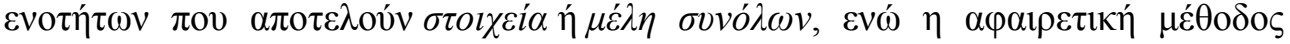

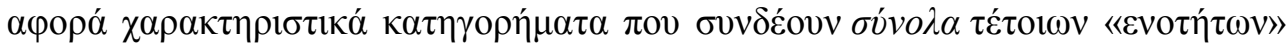

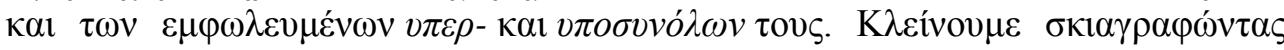

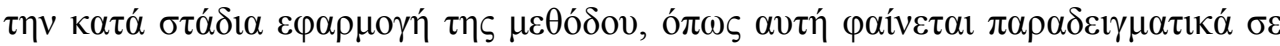

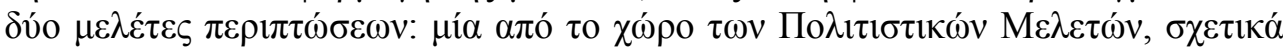

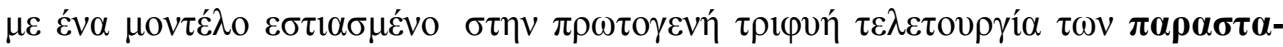

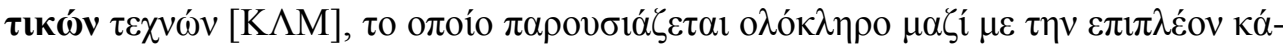

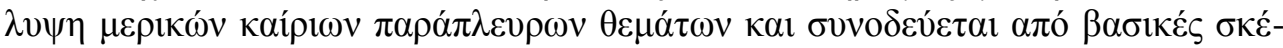

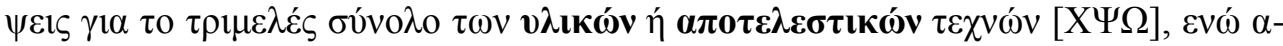

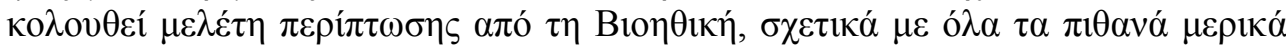

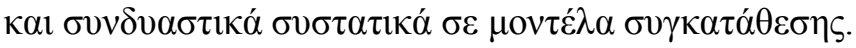

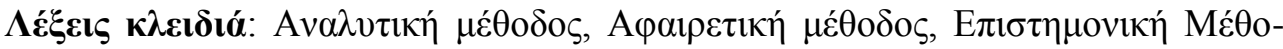

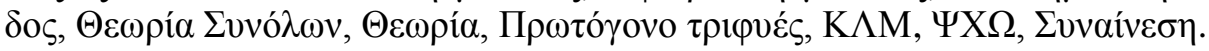

\section{Introduction}

The analytic method is a key constituent in the overall methodology of episteme $\bar{e}-$

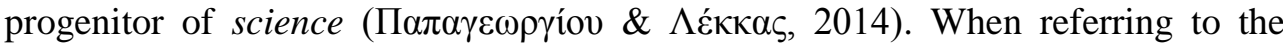
scientific method, what comes to mind is the "experimental method". But is that, or indeed, should that really be the principal methodology of science? There cannot be any experiment without bonds to a theory, all grave complications regarding the ambiguous semantics between Greek (abstract) $\theta \varepsilon \omega \rho i \alpha(t h e \bar{o} r i \bar{a})$ and Latin-derived 
(concrete, empiricist) theory/-ies notwithstanding, because those two are NOT the same thing. Other than that, experiments are doubly theory-laden: experiments in fact test "theories"; the very structure of the tested theory dictates the experimental procedure at least partly. All the other parts of the experiment, observations of its outcome included, are also dictated by still other theories - most of them taken for granted (a ceteris paribus clause is mandatory in order to ensure a controllable environment). Therefore, the main focus of the epistemonic methodological toolbox essentially underlying the scientific methodology cannot be the experimental procedure; that can only be the focus of laboratory staff. The epicentre of science can only be related to the association to theory proper and elaboration in and towards theories; that is what the analytic method and its companion counterparts are initially all about.

Theory, in the original and proper sense of the term, is an abstract construct, obtained no matter how, subject to certain criteria and freely used as an a priori organizing, describing, explaining, predicting and modeling factor for reality, if deemed to be an appropriate such factor and to the extent that it is and by the people who have agreed to accept it as such and for the realities concerned, which are then treated as an applied field of this applicable theory. If, in language and in common intuition, there is an antonymic distinction between theory and practice, then what else is the empirical observational scientific method if not an overview of "reality", and what is "reality" after all, especially as the source and depot of observational science? Is it theory or is it practice? Are observations and experiments theory, or are they practice in performance?

The overall epistemonic method puts theory proper in an external and preceding relationship to phenomena and uses it as a source of proposed and attempted screenplays, so to speak. If something fails, it is not the theory that is to blame, but a "mismatch" between phenomenon studied and theory employed or "developed", as the criteria for theory are external. What do we mean by this? For example, there are no observations of anything conceivable that could render the theoretical operation of exponentiation obsolete or, worse, "proven wrong"; misapplied maybe, useless in the situation at hand perhaps, but wrong no! Other than that, a pure theoretical epistemonic method or constituent methodological tool / "sub-method", in its constitutional status, and if it is to be of any use, ought to be essentially logical, necessarily consistent, as complete as possible, precise, clear-cut, as compact within itself as we can make it and maximally theoretically productive: the more the better. Its fitness for application is to be judged solely on the ground of its interpreting and modeling (and predicting) effectiveness. As for the logic employed, we stand by a principle that it ought to be everything we know about pure rigorous formal logic, but with three added desired attributes at its core: that it had better be clear and clear-cut and sure, that it had better be as unique as feasible (i.e. not be multi-faceted, multiple and alternative) and that it had better never challenge intuition to the point of annulment (i.e. be counter-intuitive or even notso-intuitive), because our mammalian brains have certain ways of analogy built 
round concepts and procedures, which have made us survive and which are always mobilized in our conception and expression, and which drive our "understanding".

If some "logic(s)" go(es) against these principles, as far as we are concerned, it simply isn't good enough, especially for science, and we simply have to try harder to do better. The telic reason - i.e. our very objective - why we even bother to do science, is to come up with good reliable trustworthy answers; if not, then we are conceivably facing a partial institutional failure. No one likes to ask a scientific question about the medication needed towards fixing the health of their child or towards securing the stability of the building they live in, just to get a "fuzzy" or "multiple-choice" answer, and a clear-cut verdict is always preferable to a gambleng one; at least so we trust. If we are to use logic towards studying "fact" and "reality", then, par excellence, logic had better make sense in the "good" sense of the words, rather than creating its own universes, sensory or otherwise, or, worse, making nonsense that would then be trying to pass as sense or, even worse, actually managing to do so upon mental attributes of self-consistency and such, especially circular and self-referential ones.

Thus, the scientific and epistemonic methods work in complementary opposite senses; to the former, the real world is an empirical depot and the scientific model is a desired result of practical models preferably working on and reflecting external and independent theoretical principle; to science / the sciences, the real world is an atempted and proposed source field of factual data manifesting themselves to our senses, "perceived, conceived and understood" in our neurons analogically, and

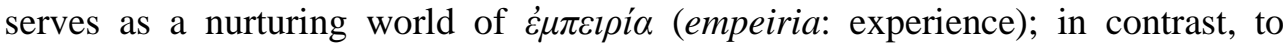
episteme, the real world is an attempted and proposed terminal field of archetypal

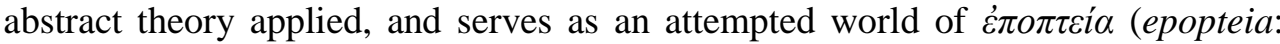
overview, manifestation).

On the organizing and real level, now, both the epistemonic method and its applied scientific counterpart make extensive use of two distinct constituent methods or submethods, both of which are twofold - at least this structure is dictated by a direct association to set theory. Now, the reason why set theory should have anything to do with the way in which science forms its methodology may only be understood to the extent that one accepts the classical mathematical "toolbox" as the desired absolute standard for abstract thought - which is, in the end, what theories are: abstract statements without a priori practically focused meaning for the world - or, equally, abstract statements with the capacity to host any meaning deemed appropriate.

A brief historical note is in order now, after which a practical illustration of the analytic method will be presented. The paper will conclude with two case studies manifesting an essential facet of the applicability of successive stages of the analytic method.

\section{Historical perspective}

As we all know, the orthodox analytic (sub-)method consists of two stages, analysis and synthesis, as a pair-wise coordinated key component of both standard 
methods: epistemonic and scientific. In order to better understand it, we should really create a frame for it and set it apart from other similar things, i.e. fulfil a task of compacting, framing and disambiguating it. The first step is to determine scopes, specify meanings and straighten out the semantics by defining in a theoretically advantageous fashion. In the beginning it looks as though the concepts might be better approached through their history, beginning in classical Greece, where we read that the various components of the analytic method were key-methods in mathematical proof. However it seems that these terms have oftentimes been used too broadly or too loosely, creating overlaps and even confusions with other methodical tools which we wish to keep separate, for reasons of rigour.

Our best bet, then, in clearing things up at the source, is, as was already mentioned above, is to define, frame and disambiguate, by first reverting to i. the dubious but potentially marginally useful aspect of etymology and ii. a careful observation of historical practice. By etymology, then, the Latin counterparts and cognates of these terms are i. $\dot{\alpha} v \alpha \dot{\lambda} \nu \sigma \sigma \varsigma($ analysis) = resolutio, breaking up (and into?) and ii. $\sigma \dot{v} v \varepsilon \sigma \sigma \varsigma$ (synthesis) $=$ compositio, putting together. The rest of ancient history, in its grand vision for quest, has not really always stayed faithful to this literal interpretation, thus operating against clarity and oftentimes pushing the terminology to overlaps and ambiguities and confusions.

"Analysis is the obtaining of the thing sought by assuming it and so reasoning up to an admitted truth; synthesis is the obtaining of the thing sought by reasoning up to the inference and proof of it" (Euclid, XIII.5 as framed by Eudoxus, Cajori 1894 p. 31). This is the oldest definition of mathematical analysis being contrasted to synthesis. Analysis was "a method of discovery, in which you consider the properties of the thing you want to find and deduce what it must be like", and synthesis was "a method of disclosure, in which you state what the result must be, without explanation, and prove you are right" (Hodgkin 2005 p. 39).

The methodology of analysis and synthesis was implicitly utilized by Hippocrates of Chios and others; but its explicit formalization was delivered no sooner than Plato's time, when he and his school "turned the instinctive logic into a conscious, legitimate method" (Cajori 1894 p. 30). Plato himself distinguishes two processes that bear striking similarities to the structural logic of analysis and synthesis. Both begin from hypotheses. The former method treats hypotheses as first principles, builds upon them and arrives at conclusions - the method of mathematics. The latter uses the hypotheses as mere stepping-stones for mounting higher and higher, until the absolute ideal and the principle of all things is reached. Here there is nothing hypothetical and one may descend again and arrive to certainties in a process that no longer needs any sensible images. This is the dialectic way (Heath 1921 pp. 290-292). Yet there still is nothing here diversifying by nature and scope of the assumption; besides, Plato's dialectic is more than analysis and synthesis, as it has elements of abstraction and structure, concepts we shall cover next. However it is in the school of Aristotle where we encounter purer forms of analysis and 
synthesis in their presently accepted sense, even though they may not be expressly stated as such.

Awareness and the specifications of the process of abstraction, on the other hand, in its clear form, is a more contemporary product of mathematicians who seem to have recently led their preference for it to a peak (Hodgin 2005 p. 236). "Bourbakists", named after N. Bourbaki (... a collective pseudonym), is a movement of the $20^{\text {th }}$ century with the intention to drive to a more abstract view of mathematics (Hodgin 2005 p. 240).

Is abstraction so powerful? Reviel Netz, in his 2003 book The Shaping of Deduction in Greek Mathematics, concludes in page 214:

"I suggest therefore that one part of the answer to "why are Greek mathematical proofs the way they are?' is that proofs are compartmentalised from broader discussions, so that their structure is wholly autonomous. When doing mathematics, one does nothing else. Instead of the multidimensional structure of interests and implications of natural discourse, Greek mathematics abstracts mathematical relationships. (...) the structure of Greek mathematical proofs is a direct, uninterrupted act of persuasion. Paradoxically, this ideal can be more perfectly achieved only when the mathematical discourse is abstracted from any context, when it is no longer part of the reallife acts of persuasion, but, rather, an artificial exercise in a compartmentalised domain. The ideal of real-life persuasion survives and reaches perfection when abstracted from its origins. Is this perhaps parallel to the developpment of the Socratic discourse, from its real-life origins, through its gradual Platonic idealisation, to its Aristotelian abstract, general reformlation?" (Netz 2003 p. 214)

The power of abstract proof is exactly the lack of external content that liberates thought from biases and ideologies that are anchored in observation. The validity of the dialectic method is confirmed not only by its accommodating capacity in regard to past observations, but almost uniquely by predictions. Zeno's paradoxes, and his abstract conception of continuum and discretum as the boundary conditions of the ad infinitum divisibility, led Democritus to the perception of atom, and foretold the modern atomic and calculus theories (see for example Heath 1921 pp. 275-238).

As synthesis is the counterpart of analysis, an analogous question arises regarding the opposite-sense counterpart of abstraction. For reasons loosely dwelling in the considerations put forth below, and understood through them, as suggested in the work of many scholars directly or indirectly, we take the responsibility of designating this counterpart as structure, intuitively at least, in the singular unambiguous sense of the Greek term $\delta o \mu \eta$ and not in any one of the other senses indiscriminately crammed into semantic confusion under the same term in

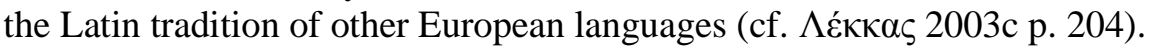

\section{Analysis - synthesis and abstraction - structure}

The modern use of the two epistemonic (sub-)methods, i.e. analytic and abstract, especially in a most notable complementary sense, can be brought to correspond to 
key developments of Set Theory that took place in the late $19^{\text {th }}$ and $20^{\text {th }}$ century. An epitome of the relevant quest and its results were concisely conceived and formulated by the second author of the present paper, in his effort to determine crucial issues regarding the arts for the of the Greek Open University; they are exposed in his relevant writings starting from the publication of the wider-scope

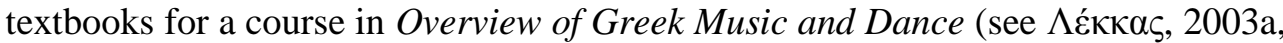
$\mathrm{b}$ and $\mathrm{c}$ ). The first thing that became clear, in association with the scientific method also here applicable to Cultural Studies, is the nature of the initial first and starting stage of observation. One essentially observes an entity and/or a procedure regarding that entity, which is deemed as one, i.e. as a single observable unit, or as a well-determined collection of such singularly identifiable entities, capable of being observed one at a time.

Thus, the concept of an observed unit / unity or clear multiplicity of identifiable unities is clear and depends on scope and on common consensus (i.e. acceptance of agreement) for those involved and agreeing to participate in the procedure and in its discussion; and then a continuum will be regarded as "one item". Regardless of how arbitrary or conventional or opportunistic this choice of focal unit may be, it becomes binding for the rest of the study for the people agreeing to accept the convention for as long as they accept it, irrelevant as it might be to others - but that is... irrelevant.

From then on, breaking up a unity, thus determined, is like dissecting an element or member of the set into constituents, whereas combining unities on the basis of the concept of categorical criteria, such as "properties" or "attributes", corresponds to collecting, grouping and forming supersets and subsets. This dichotomy is elementary and sine qua non in set theory, whereas scientists and theorists do not seem to have introduced its incumbent distinction in the epistemonic and scientific methods, or at least have been conscious of it; curiously enough, the first place where it has appeared and analyzed are our second author's said prior recent writings. According to the pathway he follows, an observable unity or unitary procedure is "one" and "synthetic / compound" by our agreement on its nature, thus digging into it and its constituents is analytic. On the other hand, these (singular) "one's", being able to be taken "one at a time", are collectible in a course of generalizing upwards developing abstraction, which can be reversed in a downward direction of more and more specification until we get back to "one" (instantiation?) and this inverse direction underlies the whole idea of structure, according to this concept. And this viewing is what dictates the operating guidelines set further on in his writings and further below here.

Set theory (or naïve set theory) is the structured approach to our intuitive (... naïve) understanding of how we make collections of "things", be they similar or not. Especially for similar objects, or objects that share at least one characteristic, we may describe the set using a criterion - thus not being obliged to describe all and every object by name. The whole development of set theory revolves round two items: sets and elements of sets. This distinction is crucial not only for mathe- 
matics - a ubiquitous staple in all axiomatic formulations ever so far - but also for our most basic understanding of the world. The distinction between elements and sets bears analogies to the distinction between things and properties (metaphysics), matter and mind (metaphysics), objects and their predicates (logic). Logic is also based on this fundamental distinction, as predicated (thus quantifiable) objects are its only non-logical elements. If we are to base our understanding of the world on logic and on set theory, we are going to use a cocktail of the analytic and abstract methods which are the outcome not only of this basic distinction, but also of the resulting calculus among set-members, sets and all their possible combinations.

One essential constituent pair of the general epistemonic (/ scientific) methodology is, then, the said analytic method. It refers to specific objects, phenomena or processes. As we already mentioned, it encompasses two stages, analysis and sy-

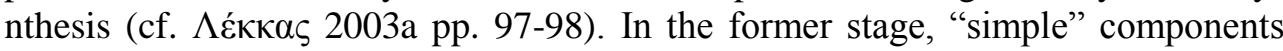
are designated (or defined) and, in the latter stage, constructions are set up, involving simple components identified in the former stage as their building blocks.

The second essential constituent pair of the epistemonic (/ scientific) methodology is the abstract method. It refers to categories of objects, phenomena and processes and to their groupings with regard to properties. These categories are also known under a different equivalent scope as predicates. Thus, a category is but a set of objects etc., subject to considerations and allowed procedures akin to the axiom of choice in set theory: each and every member of the set is an ideal representative / example, i.e. typical, sufficient and equivalent, in the sense that any representative / example is as good as any other. Properties, then, are but criteria of inclusion in such sets. The former essentially generalizing stage of the abstractive method is abstraction and thus, by mere semantics and methodological directionnality, the latter essentially specializing / specifying one comes out to be structure. Just as in the previous pair, abstraction is a process of forming or conceiving nested series of supersets, and structure is the mental conception of objects by means of their membership in overlying categories by nested inclusion or, something equivalent, the spotting of subcategories all the way to particular objects viewed as singletons, i.e. one-element sets, fully bearing the particular qualities or properties on the bottom step of the ladder of a set level: a set containing a single element carrying all desired properties and attributes or designed and built so as to carry them. In all cases, one contrasting feature between the abstract and analytic directions ought to be and stay absolutely clear, though the scientific approach has consistently and persistently been failing to mention or even conceive of this ultraelementary distinction:

$$
x \neq\{x\}
$$

In this fashion, analysis is the breakup of an entity, or member / element, into its particular components, and synthesis is the "return" from components back to the element / entity. By the same token, abstraction is the course from a particular compact entity to more and more general categories, and structure is the "return" 
from general categories towards entities they contain, each fully exhibiting the said categorical features.

It is obvious now that, however similar these two processes are and irrespective of the fact that they share one and the same starting point, they diverge towards opposite directions. Thus, the ensuing danger is grave: to confuse these two methods, as in confusing elements, alias members, and singletons, therefore sets; and, thence, to classify things based on properties, only to treat them as differing instrumentally - which is not necessarily the same: not at all. The myth of Frankenstein embodies the collective subconscious anxiety of how to structure a collection of parts - structure, life in this case, is a property, an epiphenomenon, not identified in a unique way with any combination of elements. Another such deviation of thought would be to kill a person (an analytic component) in order to punish a nation (an abstract-structural entity), as some terrorists attempt when they capture live soldiers on missions.

The discussion immediately following stems directly from the preceding development. Two facts ought to be obvious already:

a. a predicate, a quality or a property, being a feature of nested supersets, is not a "content", but indeed a container, because it is the marker of a superset, not of a subset;

b. synthesis and structure are two procedures of opposite senses, as they both constitute returns to the entity / singleton and/or its unique element (member), except that synthesis returns from the parts to the whole, i.e. it is an "ascending" return as though "from within", whereas structure returns from the graded generality of nested supersets through a few identifiable specific cases, subset by subset, all the way to the specific case of one entity / set element (member of a singleton), i.e. it is a "descending" return as though "from above".

Consequent rules of thumb regarding directionality of procedure:

$\checkmark$ no analysis without synthesis, no synthesis without analysis, no synthesis prior to completion of analysis;

$\checkmark$ no abstraction without structure, no structure without abstraction, no structure prior to completion of abstraction;

$\checkmark$ analysis and abstraction run divergently towards the sides in mutually opposite senses (away from the unitary entity); similarly, synthesis and structure run convergently from the sides towards a common point, i.e. towards the unitary entity) in mutually opposite senses. Incidentally, in consequence, i. analysis and structure run in the same sense, ii. synthesis and abstraction run in the same sense;

$\checkmark$ technically, a combination of $n$ items is not analytically or abstractly to be broken up into $n$ individual cases (constituents or members) at once, but ought to be gradually driven there by distinct successive identifiable stages, in a power-set style, as follows:

$\circ 1$ combination of $n$ items / " $n$-tuple", 
○ $n$ combinations of $(n-1)$ items / " $(n-1)$-tuples",

$\circ n(n-1) / 2$ combinations of ( $n-2)$ items / "( $n-2)$-tuples",

○ $n(n-1)(n-2) / 6$ combinations of $(n-3)$ items / "( $n-3)$-tuples",

...

$\circ n(n-1)(n-2) / 6$ triples / combinations of 3 items / triples,

$\circ n(n-1) / 2$ pairs / combinations of 2 items / pairs,

$\circ n$ singular individual items.

Similarly, in the opposite sense, a statement of a combination of $n$ individual cases of items (constituents or members) into "whole" multiplicities is not to be synthetically or structurally fused into one full collection at once, but ought to be gradually built up by distinct successive stages (pairs, triples, quadruples and so on, the same as the ones right above, in the directly opposite order).

Note that, as abstraction proceeds upwards to wider and wider containing supersets, successive slices of particular "features" of members / examples keep being dropped from the image; in other words, more and more blocks of semantic content are erased. In this sense, abstraction is a process of gradually diminishing the signified of each entity by its joint inclusion with other entities. At the end of the road, as soon as one has reached "absolute abstraction", all one is left with is void signifiers with no signified, "totally abstract" concepts / entities / "ideas", whether nameless, symbolic or named in some dummy meta-language. Mathematics is the one branch of the epistemonic method dealing in absolute abstraction - cf. what was said above about both the dialectic way disposing of the need for sensible images and about abstraction's riddance from any context (again, Heath 1921 pp. 290-292 and Netz 2003 p. 214).

Something else ought to be noted here most emphatically, as a matter of essential complementary principle, regarding the nature of both "other ends" of the respective methodological ranges. These "other (far) ends", lying on the opposite sides of i. the "elementary" analytical components of a unity at the "bottom" of an analysis and ii. of the "all- inclusive" / "most general" / "universal" categories or attributes or predicates or properties or criteria at the "far end" of the nested supersets of an abstraction, are a matter of a consensus of mutually agreed and accepted conventions among the parties concerned too.

Is there a danger when one attempts to follow the abstract method to its extreme? The greatest risk of abstract thinking comes when one deviates from mathematical reasoning and starts to daydream relentlessly. There is no danger in the alleged uncertainty / relativity of clear thought. The most absolute theoretical discipline, mathematics, is the only thoroughly abstract one throughout, in stark contrast to everything bound on experience. However, if science is not permeated by plausible mathematical quadripolar logic from end to end, one could end up daydreaming. For "daydreaming", i.e. emotionally charged reasoning (when emotions are untrained), see (Papageorgiou 2018). 
The boundary between scientific contemplation and daydreaming may turn into ultra-thin specula if short of proper restrictions.

Last but not least let, us note that the mutually different nature of the analytic and abstract sub-methods freely allows for the full number of combinatory overlaps, interfaces and dualities, i.e. i. (component-orientated) analytic abstraction and synthetic abstraction and analytic structure and synthetic structure, as well as ii. (attribute- or group- or property-orientated) abstract analysis and structural analysis and abstract synthesis and structural synthesis. The two illustrative case studies following are both typical instances of one such vital cross between the two constituent (sub-)methods, because the analysis and synthesis in both are based on (whether acknowledged or desired, whether observed or hypothesized / proposed / projected) attributes that are abstractly and/or structurally theoretical.

\section{Case study 1: the primitive ritual KAM \\ (and its material counterpart $\Psi \mathrm{X} \Omega$ )}

In our quest for a rigorous epistemonic description and classification of the fine arts, we are often typically trapped in a viewing that starts from (allegedly) "singular arts", not well defined in a "self-evident" or conventional statement of what is a single / simple self-contained fine art and what is not, starting from the classical points of view of aesthetics etc. and working synthetically towards combinations. However, this approach leaves much to be desired in the realm of criteria and procedures as regards consistency, completeness and rigour. In working towards the said textbook, the second author of this paper decided to take an alternative route suggested by the British philosophers of the late $19^{\text {th }}$ century, talking about the performing arts as splinters that have spun off an original primeval primitive ritual. According to this theoretical description, arts such as music, song, dance, poetry and drama have developed as more specialized manifestations of that ritual; and that is a unified one, a "unity", critically seen, from a point of view that may even be dismissed in a derogatory sense as primitive, profane, pagan, superstitious, ignorant, uncivilized, crude, backward, vulgar and perhaps sad and instigated by fear. Most typically, David Hume has extensively written about (and against) this ritual and its legacy (search and see, e.g., a number of relevant excerpts in Hume 1889 and elsewhere).

In using the filters of the analytic method, as applied to the rich primitive history and its historical / archaeological relics, one may see that, indeed, the primitive ritual performance is not the final constructively built product in need of explanation, but the starting point in need of analysis. This makes our integrated approach not only different from the one that is standard in Western science, but its opposite indeed - even though we owe to them the identification of the primitive ritual. This also makes our approach much more productive and much more capable of explaining the whole process, predictive and predicting various interesting levels of the process leading from a primitive ritual to a modern performance. 
A next logical step is the distinction between more compound and simpler fine arts, coming down as inherited and emancipated specialized manifestations of splinters or aspects of the original primeval ritual, which existed in it as components; and here one cannot be careful enough in setting apart a singular fine art (e.g. music) from the corresponding artistic (e.g. musical) aspect and component of something more compound, as, e.g., of opera; therefore, methodical analysis must be precise and concise both in the successive systemic stages of analytical fragmentation and also in the terminology applied so as to disambiguate rather that sinking deeper and deeper in confusion.

Immediately following, comes a next spontaneous quest about all other simple and compound arts: where do they belong? Soon it becomes apparent that the entire rest of the arts, i.e. those that do not rely on performance, are potential contributors to the ritual, in that they can ritually form the physical environment, or "format the space", in which the performing ritual can take place. What is important, here, is the similar self-righteous theoretical possibility for an analytical setup, complete with consciousness of the differences. In any case, another very fruitful realization directly following is that what sets arts apart, deep down and under, next to the artistic scope of the process of the artist, is the material makeup of the product / work of art; this line of reasoning also sets a question running as to what the material nature and resulting identifying marker of a "work" of music, dance or poetry is; this question has been mathematically resolved by the second author of the present paper, as an outcome i. of the right question being asked so that it begs and has a good chance of begetting an answer and ii. of the arsenal of modern mathematics - but that is an issue outside our present scope.

What is amazing, indeed, is the realization that the two parallel and cooperating ternary primeval ritual processes are set apart in a first dichotomy by the fact that, while the ternary performing ritual is manifested in artistic activities known as performances, further specialized as having designers and performers, the other triple, separately manifesting itself in the autonomous arts of painting, sculpture and architecture / landscaping, produces works of art that are material bodies or constructs and has no interpreters or any other kind of mediators. Dealing with the parity between these two triples, however, should not obfuscate their differences, a couple of which will be discussed along the way. Let us remark a first capital difference here already. Much as the material and environmental arts do contribute to the ritual, they are not derived from it and do not originate in it, but in the secular arena; therefore, their relationship to the ritual is, so to speak, circumstantial but not essential, or, better, "casual, not causal" - which is conceivably why the British philosophers raised no point about them.

After doing all the work on his own, the second author of the present paper spotted an identical dual aspect of two triads involving a ternary ritual and its equally ternary material / environmental counterpart in a finished form; and, apparently, that realization became popular in the West early in the $20^{\text {th }}$ century, with the widespread impact of a staple book of research on ancient music theory by 
illustrious scholar Rudolph Westphal, who cooperated with philosopher August Roßbach. Their relevant analysis and exposition was based on a volume of notes from the Lyceum and its lectures, edited and published in the $2^{\text {nd }}$ century b.c.e., by a Cretan scholar named Luci(l)us of Tarrha; incidentally, Tarrha was a city at the Eastern flank of the exit from Samaria gorge, province of Sfakia, district of Chania, Crete. In it they exhibit a dual triplicate scheme of i. material arts quoted from Luci(l)us, aptly called "arts of a finished product" ( $\left.\varepsilon^{\prime} \chi v \alpha l \alpha \dot{\alpha} \pi \circ \tau \varepsilon \lambda \varepsilon \sigma \tau \imath \kappa \alpha i\right)$, operating on the monitoring abstract criterion of symmetry $(\sigma v \mu \mu \varepsilon \tau \rho i \alpha)$, and ii. of performing arts, aptly called "arts of action" ( $\tau \dot{\varepsilon} \chi v \alpha l \pi \rho \alpha \kappa \tau l \kappa \alpha i)$, operating on the monitoring abstract criterion of rhythm ( $\dot{v} \theta \mu \dot{o} \varsigma)$ (Westphal 2017 p. 6); Aristotle and/or his associates had also devised an interesting regulatory philosophical combinatorial duality involving objectivity and subjectivity.

In any case, what emerges as really amazing, and as an indicator of the advanced level of classical Greek philosophy in its maturity, is that the main analytically superlative pattern of these thoughts had already been made in the space of Aristotle's school, missing only in the further distinctive steps we have taken, i.e. i. in the mathematical identification of the "work of a performing art" ii. in recognizing and laying out the essential successive stages of an analytic breakdown and synthetic re-emergence of its splinters, iii. in setting apart the opposing senses of analytic breakdown and synthetic (re-)integration, including devising criteria for telling them apart, iv. in the analytic terminological "cleanup" for disambiguating between integral autonomous arts and artistic components.

MATERIAL ARTS

\begin{tabular}{llll}
\hline a. Objective & $\|$ & Plastic $(\rightarrow$ sculpture $)$ & Orchestic $(\rightarrow$ dance $)$ \\
b. Subjective & $\|$ & Architecture & Harmonic $(\rightarrow$ music $)$ \\
c. Objective-subjective $\|$ & Colouring $(\rightarrow$ painting $)$ & Poetry \\
\hline
\end{tabular}

SYMMETRY RHYTHM

Another "side-effect" success of the application of the analytic method in the primitive ritual is the identification of the work of art. Indeed, it is all too easy to identify the work of art when it comes to sculpture, architecture or painting. But what about music, poetry and dance? Is the work of art the specific interpretation of, say, a musical piece? Is it the score? Is a lost score that has never been played a work of art (yes it is!)?

Through the analytic method one is able to say that a work of a performing art essentially constitutes a set of all possible performances: ones that have or have not been carried out in the past, and ones that shall or shall not be carried out in the future - each and every one of the specific performances being equivalent to all others. The work of art, is therefore, based on the axiom of choice, an equivalence class of all performances, future and past, actual and potential.

The following analytically rigorous and complete presentation of the analytic / synthetic model and its stages regards our finished product. It was first sketched in the Open University textbook we referred to ( $\check{\varepsilon} \kappa \kappa a \varsigma, 2003 \mathrm{~b}$ ), then completed in 
an unpublished explanatory letter to the students and finally published complete for the first time in the present form in a recent collective volume in Greek, attempting a coordinative approach towards a structural theory of art (which is what its title means), including a fuller meticulous parallel coverage of both triples $\mathrm{K} \Lambda \mathrm{M}$ and $\Psi \mathrm{X} \Omega$

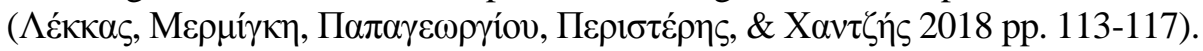

According to this new approach, a senary analytic and synthetic duality of two parallel ternary artistic complexes is sketched in the figure, followed by a full analytic and synthetic focused coverage of the ternary primitive performing ritual $\mathrm{K} \Lambda \mathrm{M}$. We deem it sufficient for its material counterpart to be presented in a sketchy incomplete suggestive manner, and here are the chief reasons why:

i. first and foremost, the single exposure of the ternary performing ritual, immediately following its joint exposure of both sets of arts in the senary (dual ternary) figure, is clear-cut, well-behaved, homogeneous, lucid, easy to follow and sufficient on its own right;

ii. as has already been cited above, in contrast to the performing arts, the material ones are "casual, not causal", as regards the primitive ritual;

iii. there are complications in the theoretical makeup surrounding the material arts, in that it is slightly but decidedly non-homogeneous, bringing complications surpassing an elementary conception and the desired eye-level clarity of perception and conception; more specifically:

a. the unity of arts of painting and sculpture, which are originally very much connected in manners far surpassing a mere complementary nature, on many levels, reaching from their artistic components of plastic / moulding and colouring - oftentimes encountered in tight synergy in their dual material combinations, often found in common artistic products of both of them (cf. coloured statues and reliefs, esp. the overpainted classical Greek sculpture, 3D tableaux), as well as in the status and artistic conscience of their creative artists (cf. the paintings of sculptor Michelangelo Buonarotti, or the sculptures of painter Pablo Picasso), as though functioning within an almost inseparable pair of "pictorial arts", taught conjointly in many fine arts schools and academies, to the point that they often appear almost inseparable (painting and sculpture often are "two arts in one packaging");

b. in contrast, the art of architecture and its supposed constituent discipline of

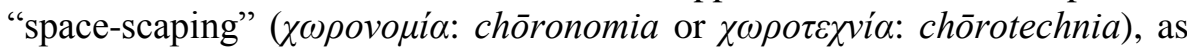
in spatial art / spatial designing, forming and constructing creative artistic disciplines, all taken together, appear multiple, manifold and diversified and are served by several specialties that are often separate, e.g. regarding buildings and all other "structures" involving statics and mechanics, components, structural and decorative and functional demands jointly and/or separately, exterior and interior spaces, and thence, in the broader sense, complexes, gardens, exterior environments, smaller or larger or extra-large constructions and monuments, landscaping etc. etc., to the point that architecture often appears almost like a practical manifold of several arts 
conjoined, and architects may be more or less generalized or specialized, much as all these directions are taught under the common educational cover of schools of architecture but may be also deemed as separable at the end of the day (architecture often is "one art in several packagings").

Moving back along our original direction, the primitive ternary ritual is a

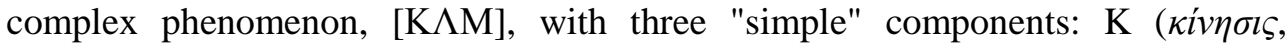

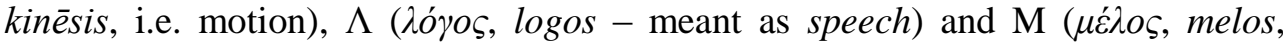
meant as the element of pitch, scale, melody, harmony and rhythm in pure music). Creatively primitive is something where all creation is due to the same person (e.g. par excellence the Greek dramatists who single-handedly wrote, composed and choreographed their dramas) and executionally primitive is what is performed by person(s) doing all, like folk improvisers of recited and sung lyrics, music and dance. In contrast, creatively posterior is something whose creation derives from the collaboration of separate creative contributors: choreographers, composers and text-writers, i.e. more specialized creative collaborators, whereas executionally posterior is a show with specialized orchestra or band musicians, singers, dancers, jugglers, actors reciting poems, each to their own specialized activity but not in common: musicians won't (necessarily) sing, singers won't (necessarily) dance, dancers won't (necessarily) recite or sing etc.

Then: A triad [K $\Lambda \mathrm{M}]$ (primary event, where $\mathrm{K}=$ motion, $\Lambda=$ speech, $\mathrm{M}=$ melos) is not immediately broken down (analyzed) into art forms consisting of a singular primitive component among these three: $[\mathrm{K}]$ (dance), $[\Lambda]$ (poetry / literature) and $[\mathrm{M}]$ (music), but first in three pairs [drama], [K $\Lambda$ ] ("fine acting") and $[\Lambda \mathrm{M}]$ (song), and then in the three components / arts: dance, poetry / literature and music, from respective pairs (the $[\mathrm{K}]$, dance, from $[\mathrm{K} \Lambda]$ and $[\mathrm{KM}]$, and so on). It is synthesized ex post in the reverse order, up to the composite $[\mathrm{K}, \Lambda, \mathrm{M}]$, designed by a choreographer, a text-writer or playwright and a music composer.

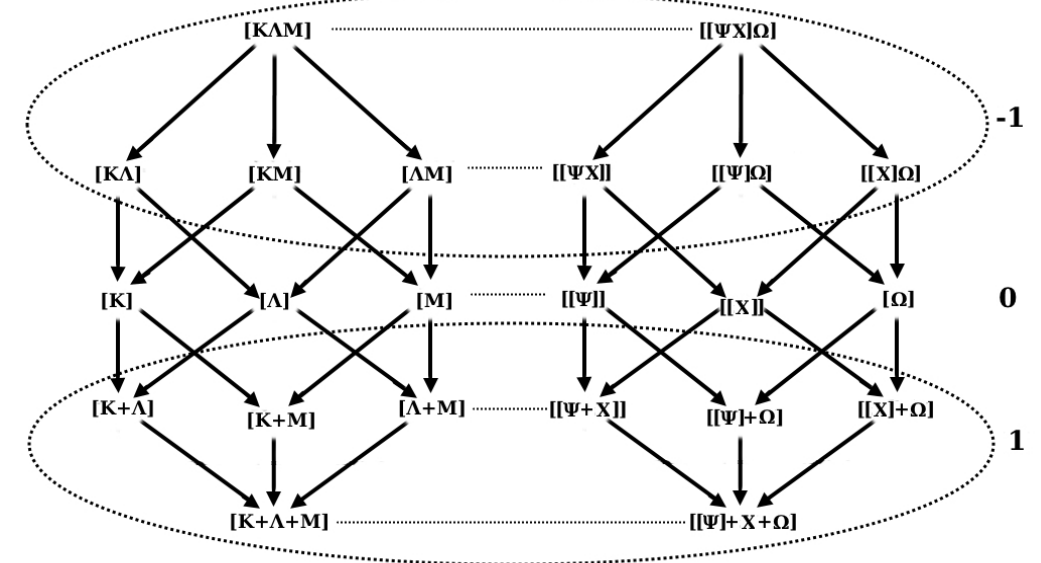

Figure 1. Models of KAM\& $\Psi X \Omega$. For acronym explanation, see text below. 


\section{A. The ternary primitive performing ritual}

$\mathrm{K}$ : motion

$\Lambda$ : speech

M: melos

$[\mathrm{K} \Lambda \mathrm{M}]:$ primitive ternary performing ritual

$[\mathrm{K} \Lambda]$ : the combination of motion and speech: drama, hypocrisis (stage acting)

$[\mathrm{KM}]$ : the combination of motion and melos: "bare" dancing to pure music (without sung text)

$[\Lambda \mathrm{M}]:$ the combination of speech and melos: song

$\mathrm{K}<[\mathrm{K} \Lambda]$ : dramatic / narrative movement, abstract dancing, general / abstract motion

$\mathrm{K}<[\mathrm{KM}]$ : dancing with specific references of form to musical accompaniment $\Lambda<[\mathrm{K} \Lambda]$ : the origin of drama: epic, dramatic and lyric poetry, prose $\Lambda<[\Lambda \mathrm{M}]$ : the origin of song: verse, poetry

$\mathrm{M}<[\mathrm{KM}]$ : pre-existing melos / instrumental music without formal speech restrictions with emphasis on the specific and abstract physical aspect (often programmatic)

$\mathrm{M}<[\Lambda \mathrm{M}]$ : the melos (tune) of the song, simple humming or vocalizing of a melodic form without words (but with a formal reference to speech)

$[\mathrm{K}]$ : orchēsis, dance (as a singular autonomous / "pure" art)

$[\Lambda]$ : cultivated speech, literature, poetry and the art of written speech, text and its recitation, read out loud

$[\mathrm{M}]:$ mousike, music, in the mature sense of the word (as a singular autonomous / "pure" art)

In the primitive ternary ritual, "music" can be anything that contains melos $\mathrm{M}$, that is, whichever one from $\mathrm{K} \Lambda \mathrm{M}, \mathrm{KM}, \Lambda \mathrm{M}, \mathrm{M}, \mathrm{K}+\mathrm{M}, \Lambda+\mathrm{M}, \mathrm{K}+\Lambda+\mathrm{M}$. One should try not to confuse the primary $(\mathrm{K} \Lambda \mathrm{M}, \mathrm{K} \Lambda, \mathrm{KM}, \Lambda \mathrm{M})$ with derivative / posterior / synthetic $(\mathrm{K}+\mathrm{L}, \mathrm{K}+\mathrm{M}, \mathrm{L}+\mathrm{M}, \mathrm{K}+\mathrm{L}+\mathrm{M})$.

\section{B. The ternary material arts complex}

To end it all, on a sketchy note, the second ternary unity, fully analysed and synthesized in the figure above but not in the subsequent text, close to the style developed according to the model set by the ternary ritual in the spirit of the analytic method and along the same principles as those set by the school of Aristotle, has the following "single" (or at least "singular") artistic components:

$\Psi:(\pi \lambda \alpha \sigma \tau \imath \kappa \eta$ : plastikē): plastic / moulding, shaping

$\mathrm{X}:(\chi \rho \omega \sigma \tau \iota \kappa \eta !$ : chrōstikē): colouring / tinting

$\Omega$ : ( $\chi \omega \rho о v o \mu i \alpha$ : chōronomiā or $\chi \omega \rho о \tau \varepsilon \chi v i \alpha$ : chōrotechniā): "space-scaping" spatial art / spatial designing, forming and constructing creative artistic disciplines (all terminology here on an attempted and arbitrary level, meant more as a trial basis on the lookout for something better, suggestions most welcome).

These are derived as definitively specifically underlying and resulting in the three acknowledged categorically autonomous material arts in the said spirit: 


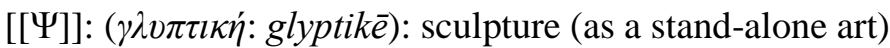

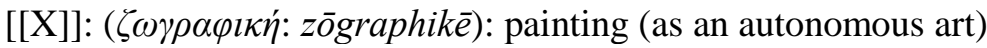

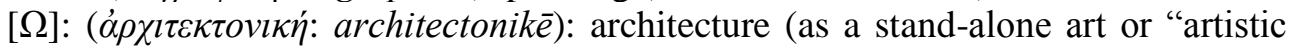
complex").

\section{Case study 2: the analytic model of consent}

The first author has used the analytic method to create a cladistic model for consent (see for example: Papageorgiou 2017b, 2017c, 2017a). Much as consent is becoming more and more important in everyday life, there is only a very limited number of types of consent, namely informed, implied, tacit and consent by proxy (Berg, Appelbaum, Lidz, \& Parker 2001; Carmi 2003; Eyal 2011; Miller, \& Wertheimer 2010). A more thorough approach is needed if one is to successfully tackle the challenges of modern society. The characteristics of our society may be said to be:

- a very complex food chain;

- production spread across the world;

- capital not concentrated in the hands of specific "capitalists", but rather fluid and spread;

- we are participating in the digital world equally to, or even more than we do in the "real" world.

How, then, should one obtain more types of consent?

One solution would be to undertake an extensive research, within the constraints of our world, and to try to find all possible types of consent one would happen to come across. After discovering them, one would name them and describe them appropriately. Apart from the obvious problem of time and resources, another big problem of this approach would be the poor categorization that would occur. Describing case after case, the order would be arbitrary and, after discovering even more types of consent, the danger of going back and replace or correct previous entries is visible - thus making the system even more chaotic.

The second solution is to discover a generator of all possible cases that would, $a$ priori, produce results valid for now, as much as for the future. That kind of generator actually exists and is none other than the rigorous stage-by-stage synthetic leg of the analytic method.

Some two hundred (200) possible variations of consent have been identified, all crucial towards understanding consent in depth and thus being able to identify, explain and make decisions about the most complex problems in our world, like bioethical problems; those, being a direct result of technological progress, would have been unthinkable, even some years before. Questions arise, having multiple implications in regard to personal freedom and choice - therefore consent -, stemming from situations such as:

- children with genetic material from three (or more!) parents;

- identifying criminals from DNA tests that their relatives have taken for medical reasons; 
- breeding animals that grow human organs.

The cladistic trees ensuing from the application of the analytic method provide an in principio and a priori estimation of the type of consent before we get to know the situation that matches any specific type of consent. Moreover, it is an inherent trait of the analytic method that it predicts all possible cases, existing or future ones. Finally, it helps towards effectively grouping the cases into coordinated categories, so there may be created equivalence classes with common ethical qualities.
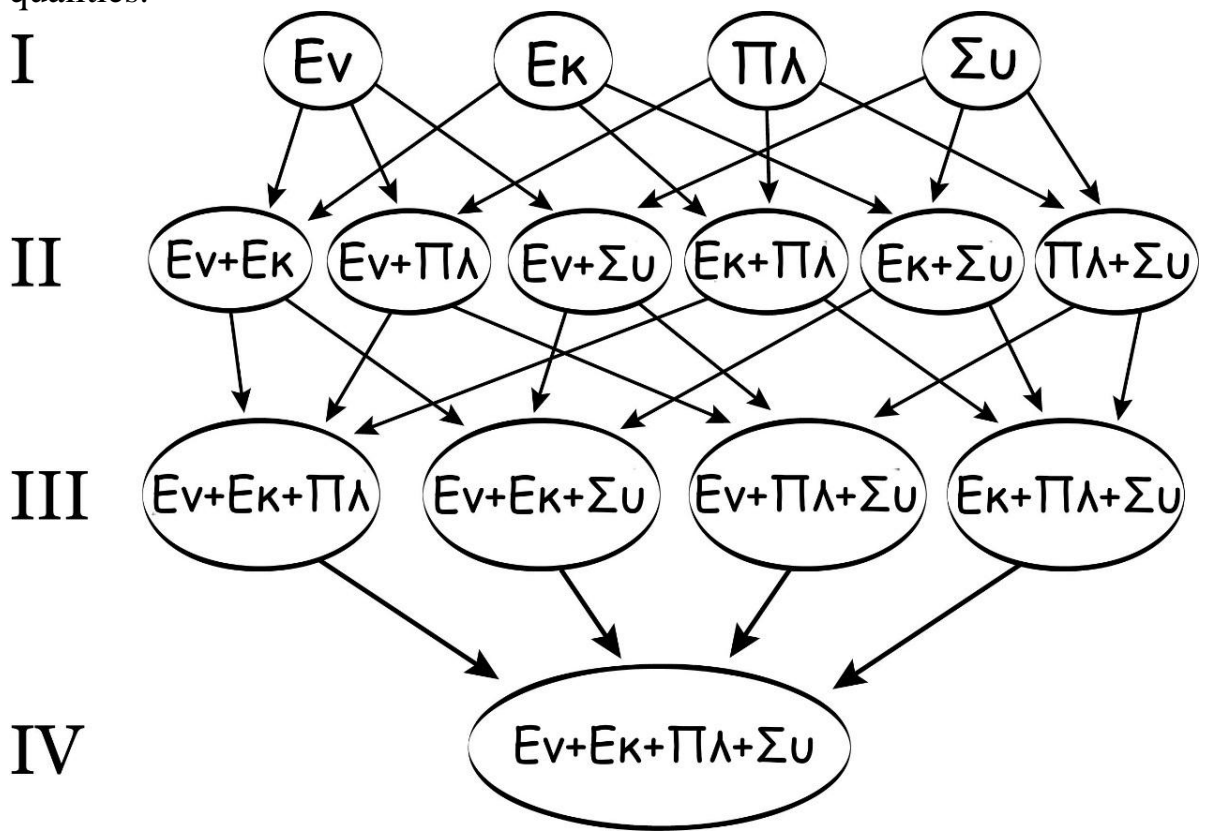

Figure 2. A cladistic tree describing informed (Ev), voluntary $(E \kappa)$, Full $(\Pi \lambda)$, consent $(\Sigma v)$.

\section{Conclusion}

In this paper we have attempted to describe the general principles, key features and thorough approach to the constitution, basic inner workings, deployment and stageby-stage breakup and assemblage of the cornerstone sub-method permeating the epistemonic method, i.e. its component known as the "analytic method", as compared and contrasted with the second cornerstone sub-method, the "abstractive method", both coming in complementary pairs running in opposite senses, i.e. analysis - synthesis, and abstraction - structure. We have also provided sketches in the direction of two case studies, i.e. two examples where the strengths of this stricter elementary formality, given in the body of the text, are made clear in both models about history and function of the arts (reconstruction and functional overview of historic facts and technical specifications) and future study (predictive layout for consent models/cases). 


\section{References}

Berg, J.W., Appelbaum, P.S., Lidz, C.W., \& Parker, L.S. (2001). Informed Consent: Legal Theory and Clinical Practice (2nd ed.). Oxford University Press.

Cajori, F. (1893). A History of Mathematics (1909th ed.). New York: The Macmillan Company.

Carmi, A. (2003). Informed Consent. (A. Carmi, Ed.). Haifa: Israel National Commission for UNESCO.

Eyal, N. (2011). Informed Consent. Retrieved from http://plato.stanford.edu/entries/informed-consent/\#IdeCon

Heath, S.T.L. (1921). A History of Greek Mathematics. Clarendon Press.

Hodgkin, L. (2005). A History of Mathematics: From Mesopotamia to Modernity. OUP Oxford.

Hume, D. (1889). The Natural History of Religion. London: A. and H. Bradlaugh Bonner. Published by eBooks Adelaide.

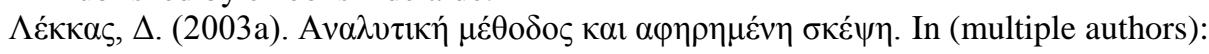

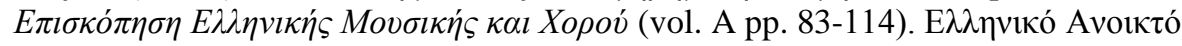

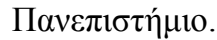

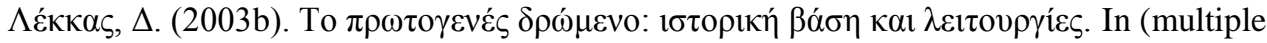

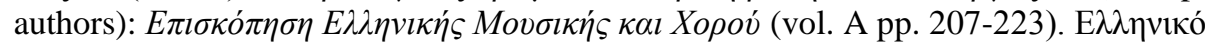

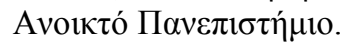

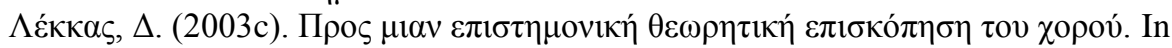

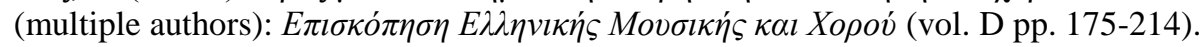

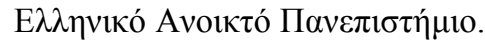

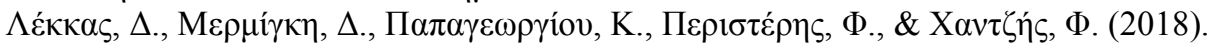

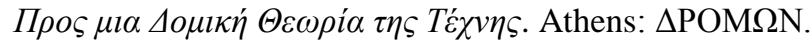

Miller, F.G., \& Wertheimer, A. (2010). The ethics of consent: theory and practice. Oxford University Press.

Netz, R. (2003). The Shaping of Deduction in Greek Mathematics: A Study in Cognitive History.

Papageorgiou, K.G. (2017a). The Subtle Colourings of (Informed) Consent in Performance Enhancement: Implications for Expertise. Philosophy Study, 7(4), 197-203.

Papageorgiou, K.G. (2017b). The Subtle Colourings of (informed) Consent in Sports Doping. In 12th World Conference Bioethics, Medical Ethics and Health Law. Limassol, Cyprus.

Papageorgiou, K.G. (2017c). Therapy, Consent and Autonomy in Perspective. In 20 years after the Oviedo Convention on Human Rights and Biomedicine. Thessaloniki, Greece: Thessaloniki Conference. Retrieved from https://www.thessaloniki-conference2017.es/

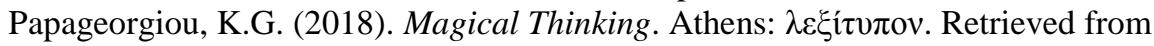
www.distalmethod.com

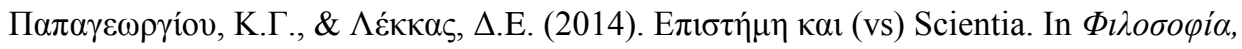

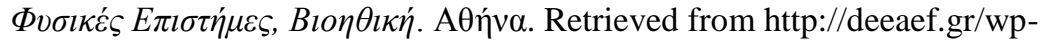
content/uploads/2016/04/Papageorgiou-Lekkas-full-text.pdf

Westphal, R. (2017). Griechische Rhythmik und Harmonik. Nicosia Cyprus: Verone P.H. Ltd., facsimile of original of 1867. 